\title{
WrightSim: Using PyCUDA to Simulate Multidimensional Spectra
}

\author{
Kyle F Sunden ${ }^{\ddagger *}$, Blaise J Thompson ${ }^{\ddagger}$, John C Wright
}

\begin{abstract}
Nonlinear multidimensional spectroscopy (MDS) is a powerful experimental technique used to interrogate complex chemical systems. MDS promises to reveal energetics, dynamics, and coupling features of and between the many quantum-mechanical states that these systems contain. In practice, simulation is typically required to connect measured MDS spectra with these microscopic physical phenomena. We present an open-source Python package, WrightSim, designed to simulate MDS. Numerical integration is used to evolve the system as it interacts with several electric fields in the course of a multidimensional experiment. This numerical approach allows WrightSim to fully account for finite pulse effects that are commonly ignored. Wright.Sim is made up of modules that can be exchanged to accommodate many different experimental setups. Simulations are defined through a Python interface that is designed to be intuitive for experimentalists and theorists alike. We report several algorithmic improvements that make Wright Sim faster than previous implementations. We demonstrated the effect of parallelizing the simulation, both with CPU multiprocessing and GPU (CUDA) multithreading. Taken together, algorithmic improvements and parallelization have made WrightSim multiple orders of magnitude faster than previous implementations. Wrightsim represents a large step towards the goal of a fast, accurate, and easy to use general purpose simulation package for multidimensional spectroscopy. To our knowledge, Wright Sim is the first openly licensed software package for these kinds of simulations. Potential further improvements are discussed.
\end{abstract}

Index Terms-Simulation, spectroscopy, PyCUDA, numerical integration, Quantum Mechanics, multidimensional

\section{Introduction}

Nonlinear multidimensional spectroscopy (MDS) is an increasingly important analytical technique for the analysis of complex chemical material systems. MDS can directly observe fundamental physics that are not possible to record in any other way. With recent advancements in lasers and optics, MDS experiments are becoming routine. Applications of MDS in semiconductor photophysics $\left[\mathrm{CTK}^{+} 15\right]$, medicine $\left[\mathrm{FGG}^{+} 09\right]$, and other domains [PLMZ18] are currently being developed. Ultimately, MDS may become a key research tool akin to multidimensional nuclear magnetic resonance spectroscopy. $\left[\mathrm{PRK}^{+}\right.$09]

A generic MDS experiment involves exciting a sample with multiple pulses of light and measuring the magnitude of the sample response (the signal). The dependence of this signal on the properties of the excitation pulses (frequency, delay, fluence,

* Corresponding author: sunden@wisc.edu

\$ University of Wisconsin--Madison

Copyright $@ 2018$ Kyle F Sunden et al. This is an open-access article distributed under the terms of the Creative Commons Attribution License, which permits unrestricted use, distribution, and reproduction in any medium, provided the original author and source are credited.

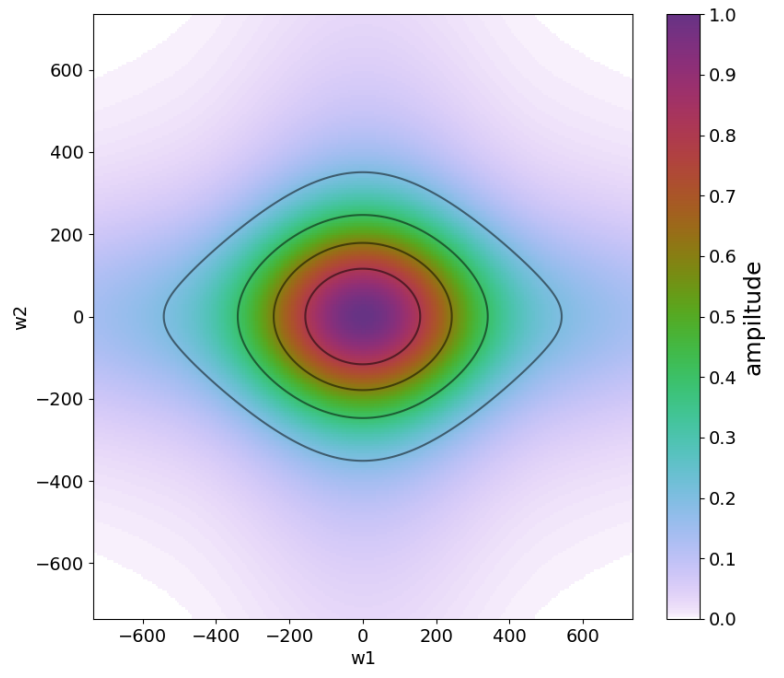

Fig. 1: Simulated spectrum at normalized coordinates

polarization etc.) contains information about the microscopic physics of the material. However, this information cannot be directly "read off" of the spectrum. Instead, MDS practitioners typically compare the measured spectrum with model spectra. A quantitative microscopic model is developed based on this comparison between experiment and theory. Here, we focus on this crucial modeling step. We present a general-purpose simulation package for MDS: WrightSim ${ }^{1}$.

Figure 1 is a visualization of a spectrum in 2-dimensional frequency-frequency space. The axes are two different frequencies for two separate input electric fields. The system that we have chosen for this simulation is very simple, with a single resonance. The axes are translated such that there is a resonance around 0.0 in both frequencies. This two-dimensional simulation is representative of WrightSim's ability to traverse through many aspects of experimental space. Every conceivable pulse parameter (delay, fluence, frequency, chirp etc.) can become an axis in the

1. Source code available at https://github.com/wright-group/WrightSim, released under MIT License. 
simulation.

WrightSim is designed with the experimentalist in mind, allowing users to parameterize their simulations in much the same way that they would collect a similar spectrum in the laboratory. WrightSim is modular and flexible. It is capable of simulating different kinds of MDS, and it is easy to extend to new kinds.

WrightSim uses a numerical integration approach that captures the full interaction between material and electric field without making common limiting assumptions. This approach makes WrightSim flexible, accurate, and interpretable. While the numerical approach we use is more accurate, it does demand significantly more computational time. We have focused on performance as a critical component of WrightSim. Here we report algorithmic improvements which have significantly decreased computational time (i.e. wall clock time) relative to prior implementations. We also discuss parallelization approaches we have taken, and show how the symmetry of the simulation can be exploited. While nascent, WrightSim has already shown itself to be a powerful tool, greatly improving execution time over prior implementation.

\section{A Brief Introduction of Relevant Quantum Mechanics}

This introduction is intended to very quickly introduce what is being done, but not why. If you are interested in a more complete description, please refer to Kohler, Thompson, and Wright. [KTW17]

WrightSim uses the density matrix formulation of quantum mechanics. This formulation allows us to describe mixed states (coherences) which are key players in light-matter-interaction and spectroscopy. This involves numerically integrating the Liouvillevon Neumann equation [Gib02]. This strategy has been described before [GED09], so we are brief in our description here.

WrightSim calculates multidimensional spectra for a given well-defined Hamiltonian. We do not make common limiting assumptions that allow reduction to analytical expressions. Instead, we propagate all of the relevant density matrix elements, including populations and coherences, in a numerical integration. This package does not perform ab initio computations. This places WrightSim at an intermediate level of theory where the Hamiltonian is known, but accurately computing the corresponding multidimensional spectrum requires complicated numerical analysis.

Now, we focus on one representative experiment and Hamiltonian. In this case, we are simulating the interactions of three electric fields to induce an output electric field. For three fields, there are $3 !=6$ possible time orderings for the pulses to interact and create superpositions or populations in the material system (Figure 2, columns). Within each time ordering, there are several different pathways (Figure 2, rows). In total, there are 16 pathways, represented in Figure 2 as a series of wave mixing energy level (WMEL) diagrams [LA85]. We are restricting this simulation to have two positive interactions (solid up arrows or dashed down arrows) and one negative interaction (dashed up arrow or solid down arrow). Experimentalists isolate this condition spatially using an aperture. They can isolate the time orderings by introducing delays between pulses. Simulation allows us to fully separate each pathway, leading to insight into the nature of pathway interference in the total signal line shape.

Figure 3 shows a finite state automaton for the same system as Figure 2. The nodes are the density matrix elements themselves.

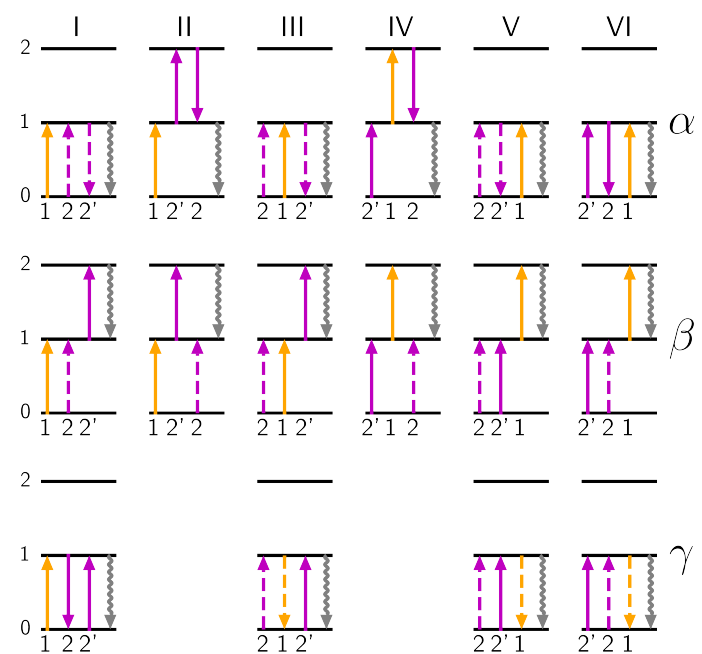

Fig. 2: Independent Liouville pathways simulated. Excitations from $\omega_{1}$ are in yellow, excitations from $\omega_{2}=\omega_{2^{\prime}}$ are shown in purple. Figure was originally published as Figure 1 of Kohler, Thompson, and Wright [KTW17]

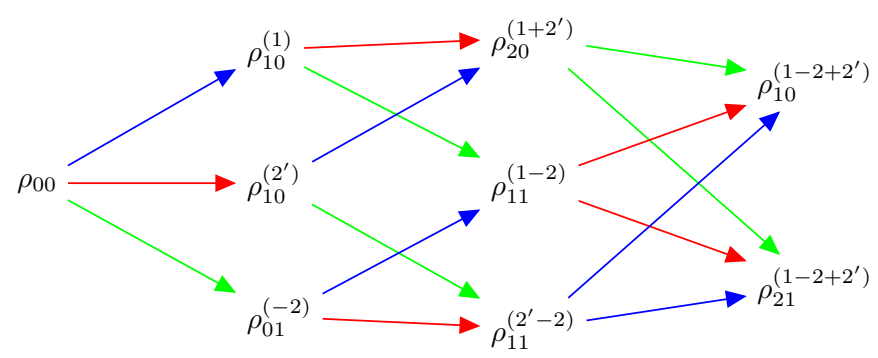

Fig. 3: Finite state automaton of the interactions with the density matrix elements. Matrix elements are denoted by their coherence/population state (the subscript) and the pulses which they have already interacted with (the superscript). Arrows indicate interactions with $\omega_{1}$ (blue), $\omega_{2^{\prime}}$ (red), and $\omega_{2}$ (green). Figure was originally published as Figure S1 of Kohler, Thompson, and Wright [KTW17]

All pathways start at the ground state $\left(\rho_{00}\right)$. Encoded within each node is both the quantum mechanical state and the fields with which the system has already interacted. Interactions occur along the arrows, which generate density in the resulting state. Here, the fields must each interact exactly once. Output is generated by the rightmost two nodes, which have interacted with all three fields. These nine states represent all possible states which match the criterion described by the process we are simulating.

We take these nine states and collect them into a state density 
vector, $\bar{\rho}$ (Equation 1.1):

$$
\bar{\rho} \equiv\left[\begin{array}{c}
\tilde{\rho}_{00} \\
\tilde{\rho}_{01}^{(-2)} \\
\tilde{\rho}_{10}^{\left(2^{\prime}\right)} \\
\tilde{\rho}_{10}^{(1)} \\
\tilde{\rho}_{20}^{\left(1+2^{\prime}\right)} \\
\tilde{\rho}_{11}^{(1-2)} \\
\tilde{\rho}_{11}^{\left(2^{\prime}-2\right)} \\
\tilde{\rho}_{10}^{\left(1-2+2^{\prime}\right)} \\
\tilde{\rho}_{21}^{\left(1-2+2^{\prime}\right)}
\end{array}\right]
$$

Next we need to describe the transitions within these states. This is the Hamiltonian matrix. Since we have nine states in our density vector, the Hamiltonian is a nine by nine matrix. To simplify representation, six time dependent variables are defined:

$$
\begin{aligned}
A_{1} & \equiv \quad \frac{i}{2} \mu_{10} e^{-i \omega_{1} \tau_{1}} c_{1}\left(t-\tau_{1}\right) e^{i\left(\omega_{1}-\omega_{10}\right) t} \\
A_{2} & \equiv \frac{i}{2} \mu_{10} e^{i \omega_{2} \tau_{2}} c_{2}\left(t-\tau_{2}\right) e^{-i\left(\omega_{2}-\omega_{10}\right) t} \\
A_{2^{\prime}} & \equiv \frac{i}{2} \mu_{10} e^{-i \omega_{2^{\prime}} \tau_{2^{\prime}}} c_{2^{\prime}}\left(t-\tau_{2^{\prime}}\right) e^{i\left(\omega_{2^{\prime}}-\omega_{10}\right) t} \\
B_{1} & \equiv \frac{i}{2} \mu_{21} e^{-i \omega_{1} \tau_{1}} c_{1}\left(t-\tau_{1}\right) e^{i\left(\omega_{1}-\omega_{21}\right) t} \\
B_{2} & \equiv \frac{i}{2} \mu_{21} e^{i \omega_{2} \tau_{2}} c_{2}\left(t-\tau_{2}\right) e^{-i\left(\omega_{2}-\omega_{21}\right) t} \\
B_{2^{\prime}} & \equiv \frac{i}{2} \mu_{21} e^{-i \omega_{2^{\prime}} \tau_{2^{\prime}}} c_{2^{\prime}}\left(t-\tau_{2^{\prime}}\right) e^{i\left(\omega_{2^{\prime}}-\omega_{21}\right) t}
\end{aligned}
$$

These variables each consist of a constant factor of $\frac{i}{2}$, a dipole moment term $\left(\mu_{10 \mid 21}\right)$, an electric field phase and amplitude (the first exponential term), an envelope function ( $c$, a Gaussian function here), and a final exponential term which captures the resonance dependence. These variables can then be used to populate the matrix:

$$
\overline{\bar{Q}} \equiv\left[\begin{array}{ccccccccc}
0 & 0 & 0 & 0 & 0 & 0 & 0 & 0 & 0 \\
-A_{2} & -\Gamma_{10} & 0 & 0 & 0 & 0 & 0 & 0 & 0 \\
A_{2^{\prime}} & 0 & -\Gamma_{10} & 0 & 0 & 0 & 0 & 0 & 0 \\
A_{1} & 0 & 0 & -\Gamma_{10} & 0 & 0 & 0 & 0 & 0 \\
0 & 0 & B_{1} & B_{2^{\prime}} & -\Gamma_{20} & 0 & 0 & 0 & 0 \\
0 & A_{1} & 0 & -A_{2} & 0 & -\Gamma_{11} & 0 & 0 & 0 \\
0 & A_{2^{\prime}} & -A_{2} & 0 & 0 & 0 & -\Gamma_{11} & 0 & 0 \\
0 & 0 & 0 & 0 & B_{2} & -2 A_{2^{\prime}} & -2 A_{1} & -\Gamma_{10} & 0 \\
0 & 0 & 0 & 0 & -A_{2} & B_{2^{\prime}} & B_{1} & 0 & -\Gamma_{21}
\end{array}\right]
$$

The $\Gamma$ values along the diagonal represent loss terms such as dephasing (loss of coherence) and population relaxation. To isolate a given time ordering, we can simply set the value of elements which do not correspond to that time ordering to zero.

At each time step, the dot product of the matrix with the $\bar{\rho}$ vector is the change in the $\bar{\rho}$ vector to the next time step (when multiplied by the differential). WrightSim uses a second order technique (Runge-Kutta) [BDH06] for determining the change in the $\bar{\rho}$ vector. The core of the simulations is to take the $\bar{\rho}$ vector and multiply by the Hamiltonian at each time step (noting that the Hamiltonian is time dependant, as are the electric fields, themselves). This process repeats over a large number of small time steps, and must be performed separately for any change in the inputs (e.g. frequency $[\omega]$ or delay $[\tau]$ ). As a result, the operation is highly parallelizable. The integration is performed in the rotating frame so the number of time steps can be as small as possible.
Usage

WrightSim is designed in a modular, extensible manner in order to be friendly to experimentalists and theorists alike. The key steps to running a basic simulation are:

- Define the experimental space

- Select a Hamiltonian for propagation

- Run the scan

- Process the results

Experimental spaces are defined in an INI format that defines a set of parameters and specifies their defaults and relationships. This can be thought of as a particular experimental setup or instrument.

We use the same experiment and Hamiltonian described above to demonstrate usage. Here, we are using a space called trive which provides, among other settings, two independent frequency axes and two independent delay axes, controlling a total of three incident pulses. The frequency axes are called $\mathrm{w} 1$ and $\mathrm{w} 2^{2}$, the delays are $\mathrm{d} 1$ and $\mathrm{d} 2$. To scan a particular axis, simply set the points array to a NumPy [?] array and set it's active attribute to True. You can also set a static value for any available axis, by setting the points attribute to a single number (and keeping active set to False). Finally, the experiment class defines the timing of the simulation. Three main parameters control this: timestep, which controls the size of each numerical integration step, early_buffer, which defines how long to integrate before the first pulse maximum, and late_buffer, which defines how long to integrate after the last pulse maximum. Here is an example of setting up a 3D (shape 64x64x32) scan with an additional static parameter set:

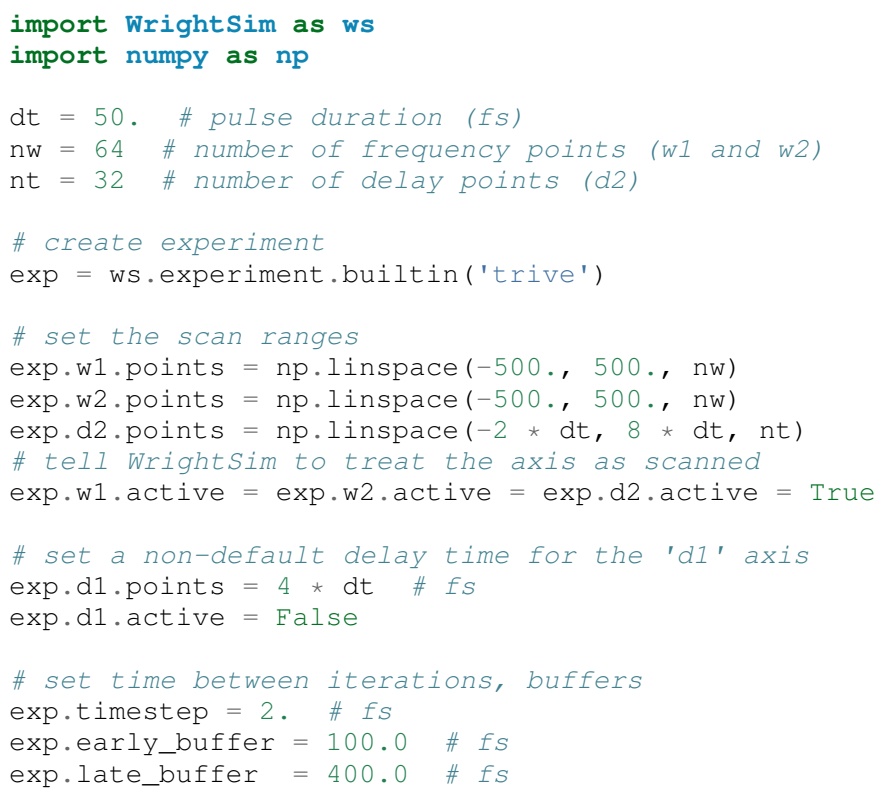

The Hamiltonian object is responsible for the density vector and holding on to the propagation function used when the experiment is run. Included in the density vector responsibility is the identity of which columns will be returned in the end result array. Hamiltonians may have arbitrary parameters to define themselves in intuitive ways. Under the hood, the Hamiltonian class also holds the $\mathrm{C}$ struct and source code for the PYCUDA implementation and a method to send itself to the CUDA device. Here is an example

2. Note, while the Latin character $w$ is used here because it is easier to type in code, it actually represents the Greek letter $\omega$, conventionally, a frequency. 
of setting up a Hamiltonian object with restricted pathways and explicitly set recorded element parameters:

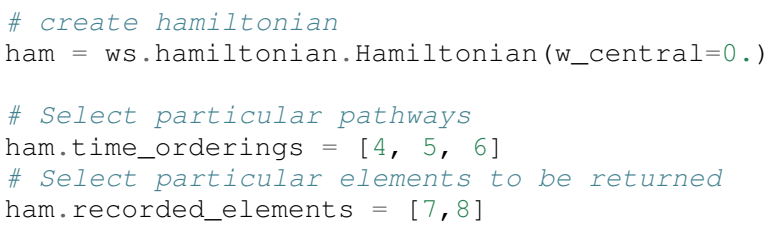

Finally, all that is left is to run the experiment itself. The run method takes the Hamiltonian object and a keyword argument mp, short for "multiprocess". Any value that evaluates to False will run non-multiprocessed (i.e. single threaded). Almost all values that evaluates to True with run CPU - multiprocessed with the number of processes determined by the number of cores of the machine. The exception is the special string 'gpu', which will cause WrightSim to run using PyCUDA.

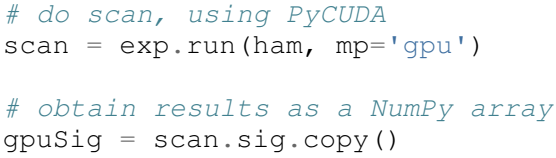

Running returns a Scan object, which contains several internal features of the scan including the electric field values themselves. The important part, however is the signal array that is generated. In this example, the complex floating point number array is of shape $(2 \times 64 \times 64 \times 32)$ (i.e. the number of recorded_elements followed by the shape of the experiment itself). These numbers can be easily manipulated and visualized to produce spectra like that seen in 1. The Wright Group also maintains a library for working with multidimensional data, WrightTools $\left[\mathrm{TSM}^{+}\right]$. This library will be integrated more fully to provide even easier access to visualization and archival storage of simulation results.

\section{Performance}

Performance is a critical consideration in the implementation of WrightSim. Careful analysis of the algorithms, identifying and measuring the bottlenecks, and working to implement strategies to avoid them are key to achieving the best performance possible. Another key is taking advantage of modern hardware for parallelization. These implementations have their advantages and trade-offs, which are quantified and examined in detail herein.

NISE [Gro16] is the package written by Kohler and Thompson while preparing their manuscript [KTW17]. NISE uses a slight variation on the technique described above, whereby they place a restriction on the time ordering represented by the matrix, and can thus use a seven element state vector rather than a 9 element state vector. This approach is mathematically equivalent to that presented above. NISE is included here as a reference for the performance of previous simulations of this kind.

\section{Algorithmic Improvements}

When first translating the code from NISE into WrightSim, we sought to understand why it took so long to compute. We used Python's standard library package cProfile to produce traces of execution, and visualized them with SnakeViz [jif17]. Figure 4 shows the trace obtained from a single-threaded run of NISE simulating a $32 \times 32 \times 16$ frequency-frequency-delay space. This trace provided some interesting insights into how the algorithm could be improved. First, $99.5 \%$ of the time is spent inside of a loop which is highly parallelizable. Second, almost one third of that time was spent in a specific function of NumPy, ix_. Further inspection of the code revealed that this function was called in the very inner most loop, but always had the same, small number of parameters. Lastly, approximately one tenth of the time was spent in a particular function called rotor (the bright orange box in Figure 4). This function computed $\cos ($ thet $a)+1 j * \sin ($ thet $a)$, which could be replaced by the equivalent, but more efficient $\exp (1 j *$ theta $)$. Additional careful analysis of the code revealed that redundant computations were being performed when generating matrices, which could be stored as variables and reused.

When implementing Wright Sim, we took into account all of these insights. We simplified the code for matrix generation and propagation by only having the one 9 by 9 element matrix rather than two 7 by 7 matrices. The function that took up almost one third the time (ix_) was removed entirely in favor of a simpler scheme for denoting which values to record, simply storing a list of the indices directly. We used variables to store the values needed for matrix generation, rather than recalculating each element. As a result, solely by algorithmic improvements, almost an order of magnitude speedup was obtained (See Figure 5). Still, 99\% of the time was spent within a highly parallelizable inner loop.

\section{CPU and GPU Parallel Implementations}

NISE already had, and WrightSim inherited, CPU multiprocessed parallelism using the Python standard library multiprocessing interface. Since almost all of the program is parallelizable, this incurs a four times speedup on a machine with four processing cores (limited more by the operating system scheduling other tasks than by Amdahl's law). This implementation required little adjustment outside of minor API tweaks.

In order to capitalize on the highly parallelizable nature of our multidimensional simulation, the algorithm was re-implemented using Nvidia CUDA [NBGS08]. In order to make the implementation as easy to use as possible, and maintainable over the lifetime of WrightSim, PyCUDA $\left[\mathrm{KPL}^{+} 12\right]$ was used to integrate the call to a CUDA kernel from within Python. PyCUDA allows the source code for the device side functions (written in $\mathrm{C} / \mathrm{C}++$ ) to exist as strings within the Python source files. These strings are just-in-time compiled (using $\mathrm{nvCC}$ ) immediately prior to calling the kernel. For the initial work with the CUDA implementation, only one Hamiltonian and one propagation function were written, however it is extensible to additional methods. The just-in-time compilation makes it easy to replace individual functions as needed (a simple form of metaprogramming).

The CUDA implementation is slightly different from the pure Python implementation. It only holds in memory the Hamiltonian matrices for the current and next step, where the Python implementation computes all of the matrices prior to entering the loop. This was done to conserve memory on the GPU. Similarly, the electric fields are computed in the loop, rather than computing all ahead of time. These two optimizations reduce the memory overhead, and allow for easier to write functions, without the help of NumPy to perform automatic broadcasting of shapes.

\section{Scaling Analysis}

Scaling analysis, tests of the amount of time taken by each simulation versus the number of points simulated, were conducted for each of the following: NISE single threaded, NISE Multiprocessed using four cores, WrightSim Single threaded, WrightSim Multiprocessed using four cores, and WrightSim CUDA implementation. A machine with an Intel Core i5-7600 


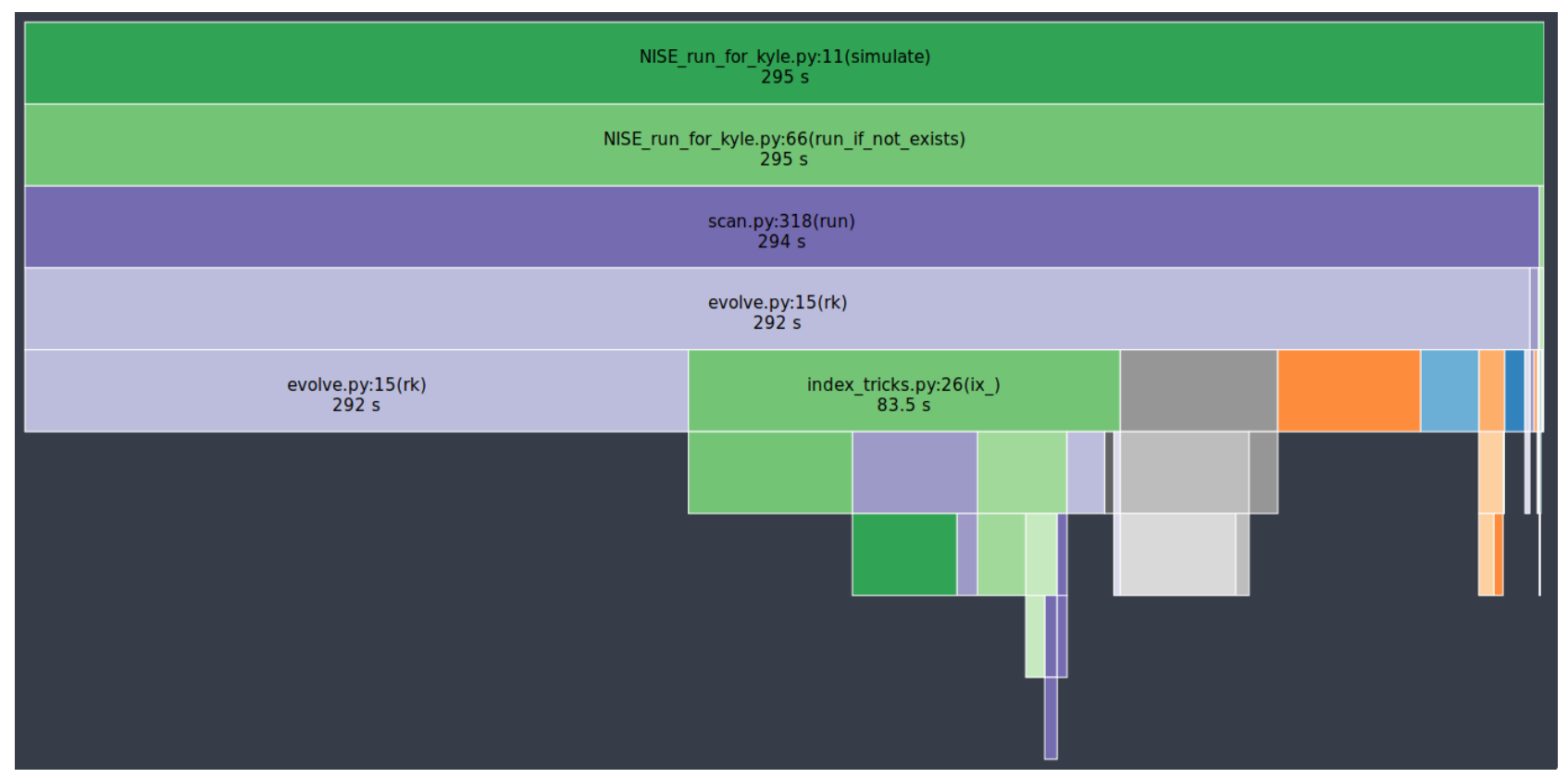

Fig. 4: Profile trace of a single threaded simulation from NISE.

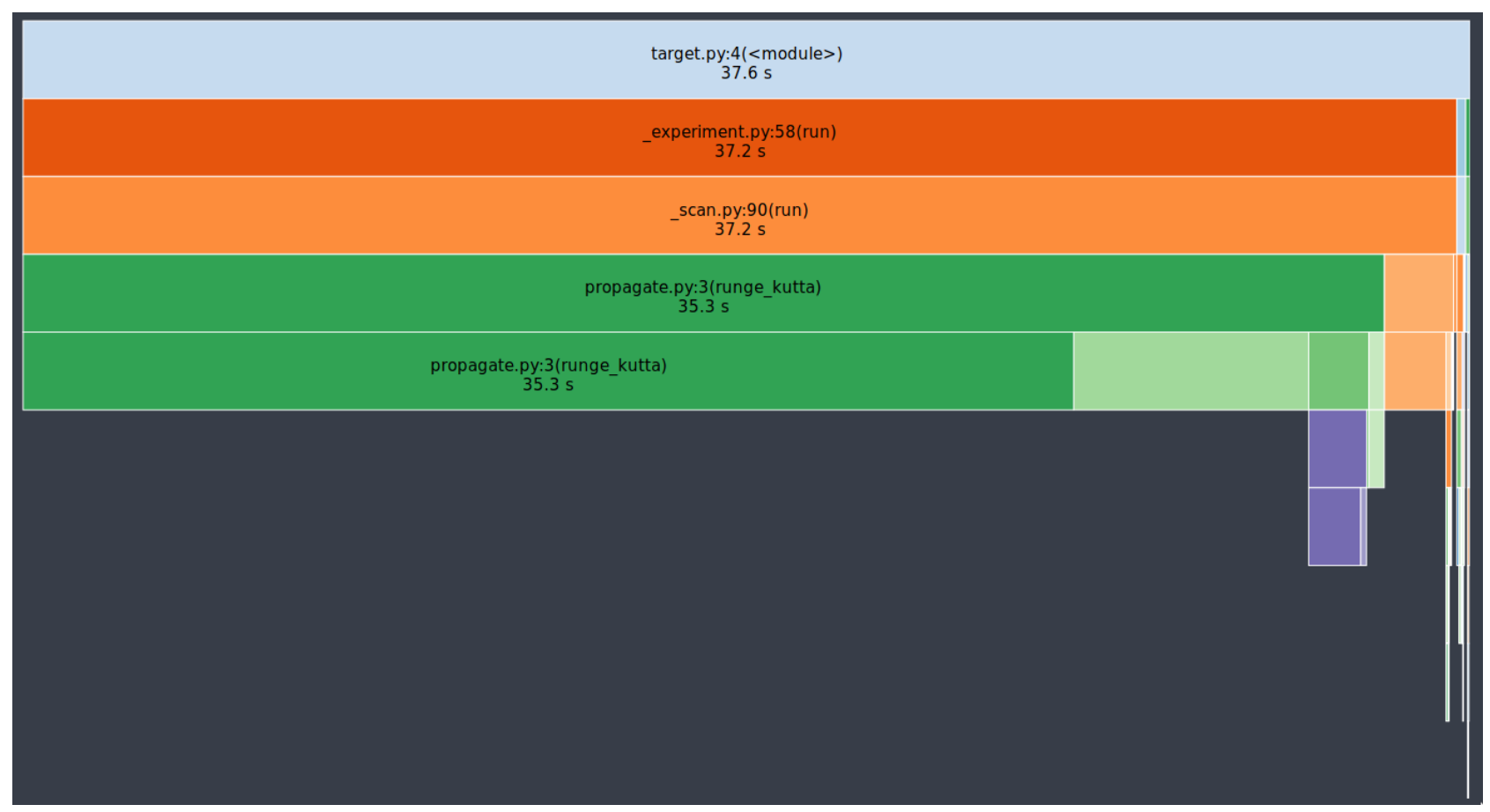

Fig. 5: Profile trace of a single threaded simulation from Wright Sim.

(3.5 GHz) CPU and an Nvidia GTX 1060 (3GB) graphics card, running Arch Linux was used for all tests. The simulations were functionally identical, with the same number of time steps and same recorded values. The NISE simulations use two seven by seven matrices for the Hamiltonian, while the WrightSim simulations use a single nine by nine matrix. The results are summarized in Figure 6.

The log-log plot shows that the time scales linearly with number of points. All lines have approximately the same slope at high values of $\mathrm{N}$, though the CUDA implementation grows slower at low N. The Algorithmic improvements alone offer doubled per- formance over even 4-Core multiprocessed NISE simulation. The CUDA implementation has a positive intercept at approximately 200 milliseconds. This is due, in large part, to the compilation overhead.

\section{Limitations}

The CUDA implementation faces limitations at both ends in terms of number of points. On the low side, the cost of compilation and transfer of data makes it slower than the 4-Core CPU Multiprocessing implementation. This crossover point is approximately 256 points (for this simulation, all other parameters being equal). Incidentally, that is also a hard coded block size for the CUDA 


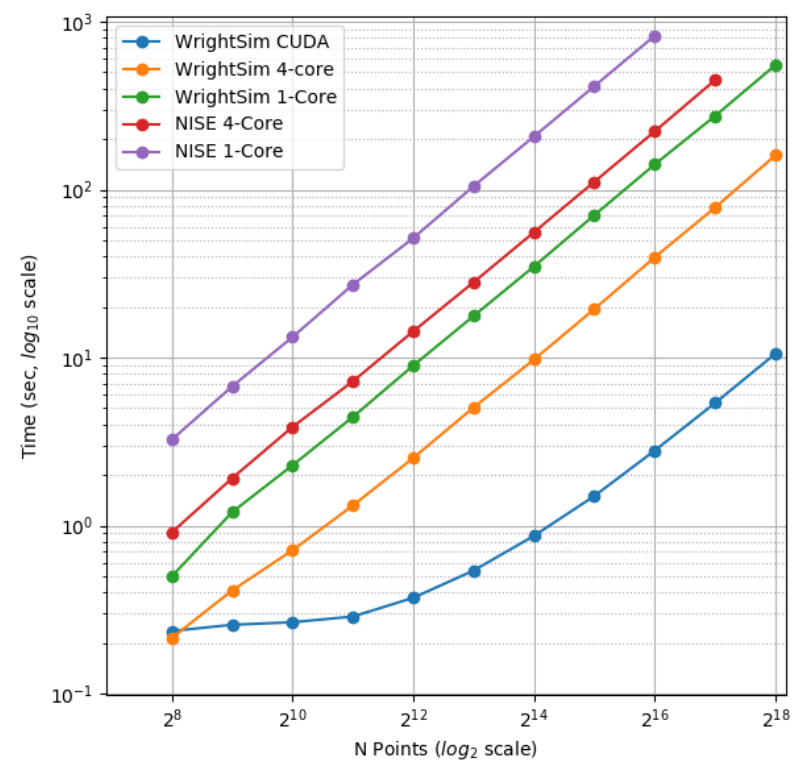

Fig. 6: Scaling Comparison of Wright Sim and NISE

kernel call. While this could be modified to ensure no illegal memory accesses occur on smaller cases, the fact that you are not saving by using CUDA (and even single core performance is under a second) means it is not worth the effort at this time. The hard-coded block size also means that multiples of 256 points must be used in the current implementation.

With larger number of points, we are limited by the amount of memory available to be allocated on the GPU. For each pixel in the simulations presented here, 250 complex numbers represented as doubles must be allocated. Additional space is needed, however it is dominated by this array, which contains the outputs which are then transferred back to the host. Each CUDA thread additionally dynamically allocates the arrays it needs to perform the computation. The current implementation, paired with the particular hardware used, has a limit somewhere between $2^{18}$ and $2^{19}$ points. This limit could be increased by using single precision floating point numbers to represent the complex arrays, if the precision trade-off is acceptable (which is yet to be determined).

\section{Future Work}

This is still quite early days for WrightSim. While it is already a promising proof of concept display of how PyCUDA can be applied to this problem, there is still much room for improvement. In general, there are improvements to be made in terms of features, API/ease of use, and indeed further algorithmic improvements.

\section{Features}

NISE had implemented a few additional features which were not carried over to Wright Sim during the initial development efforts which focused on performance thus far.

There was support for chirped electric field pulses, which behave in less ideal fashions than the true sinusoids and Gaussian peaks used thus far. These non-ideal perturbations can have a real effect in spectra collected in the lab, and accurately modelling them helps to interpret these spectra.
Samples in laboratory experiments may have some amount of inhomogeneity within the sample, resulting in broader than would otherwise be expected peaks. This inhomogeneity can be modeled by storing the response array which is calculated by numerical integration, and translating the points slightly. The original NISE implementation would perform the simulation multiple times, where that is not needed as a simple translation will do. At one point we considered generating a library of responses in well known coordinates and saving them for future use, avoiding the expensive calculation all together. That seems to be less urgent, given the speed of the CUDA code.

NISE provided a powerful and flexible set of tools to "Measure" the signal, using Fourier transforms and produce arrays that even further mimic what is observed experimentally. That system needs to be added to WrightSim for it to be feature-complete. More naïve methods of visualizing work in this case, but a true measurement would allow for richer, more detailed analysis and interpretation.

Some new features could be added, including saving intermediate responses using an HDF5 based file format. The CUDA implementation itself would benefit from some way of saving the compiled code for multiple runs, removing the 0.2 second overhead. Current implementation compiles directly before calling the kernel, whether it has compiled it before or not. If performing many simulations in quick succession (e.g. a simulation larger than the memory allows in a single kernel call) with the same $\mathrm{C}$ code, the savings would add up.

The just-in-time compilation enables some special metaprogramming techniques which could be explored. The simple case is using separately programmed functions which have the same signature to do tasks in different ways. Currently there is a small shortcut in the propagation function which uses statically allocated arrays and pointers to those arrays rather than using dynamically allocated arrays. This relies on knowing the size at compilation time. The numbers could be replaced by preprocessor macros which are also fed to the compiler to assign this value "pseudo-dynamically" at compilation time. A much more advanced metaprogramming technique could, theoretically, generate the $\mathrm{C}$ struct and Hamiltonian generation function by inspecting the Python code and performing a translation. Such a technique would mean that new Hamiltonians would only have to be implemented once, in Python, and users who do not know $\mathrm{C}$ would be able to run CUDA code.

\section{Usability}

One of the primary reasons for reimplementing the simulation package is to really think about our interface. As much as possible, the end user should not need to be an experienced programmer to be able to get a simulation. One of the next steps for Wright Sim is to take a step back and ensure that our API is sensible and easy to follow. We wish to, as much as possible, provide ways of communicating through configuration files, rather than code. Ultimately, a GUI front end may be desirable, especially as the target audience is primarily experimentalists.

Additional Hamiltonians would make the package significantly more valuable as well. To add more Hamiltonians will require ensuring the code is robust, that values are transferred as expected. A few small assumptions were made in the interest of efficiency in the original implementation. Certain values, such as the initial density vector, represented by the Hamiltonian were hard-coded on the device code. While the hard-coded values are reasonable 
for most simulations, the ability to set theses at run time is desired, and will be added in the future.

\section{Further Algorithmic Improvements}

While great strides were taken in improving the algorithms from previous implementations, there are several remaining avenues to gain improved performance in execution time and memory usage. The CUDA implementation is memory bound, both in terms of what can be dispatched, and in terms of time of execution. The use of single precision complex numbers (and other floating point values) would save roughly half of the space. One of the inputs is a large array with parameters for the each electric field at each pixel. This array contains much redundant data, which could be compressed with the parsing done in parallel on the device.

If the computed values could be streamed out of the GPU once computed, while others use the freed space, then there would be almost no limit on the number of points. This relies on the ability to stream data back while computation is still going, which we do not have experience doing, and are not sure CUDA even supports. The values are not needed once they are recorded, so there is no need from the device side to keep the values around until computation is complete.

Additional memory could be conserved by using a bit field instead of an array of chars for determining which time orderings are used as a boolean array. This is relatively minimal, but is a current waste of bits. The Python implementation could potentially see a slight performance bump from using a boolean array rather than doing list searches for this same purpose.

The CUDA implementation does not currently take full advantage of shared cache. Most of the data needed is completely separated, but there are still a few areas where it could be useful.

The current CUDA implementation fills the Hamiltonian with zeros at every time step. The values which are nonzero after the first call are always going to be overwritten anyway, so this wastes time inside of of nested loop. This zeroing could be done only before the first call, removing the nested loop. Additionally, many matrices have a lot of zero values. Often they are triangular matrices, which would allow for a more optimized dot product computation which ignores the zeros in the half which is not populated. Some matrices could even benefit by being represented as sparse matrices, though these are more difficult to use.

Finally, perhaps the biggest, but also most challenging, remaining possible improvement would be to capitalize on the larger symmetries of the system. It's a non-trivial task to know which axes are symmetric, but if it could be done, the amount that actually needs to be simulated would be much smaller. Take the simulation in Figure 1. This was computed as it is displayed, but there are two orthogonal axes of symmetry, which would cut the amount actually needed to replicate the spectrum down by a factor of four. Higher dimensional scans with similar symmetries would benefit even more.

\section{Conclusions}

WrightSim, as implemented today, represents the first major step towards a cohesive, easy to use, fast simulation suite for quantum mechanical numerically integrated simulations using density matrix theory. Solely algorithmic improvements enabled the pure Python implementation to be an order of magnitude faster than the previous implementation. The algorithm is highly parallelizable, enabling easy CPU level parallelism. A new implementation provides further improvement than the CPU parallel code, taking advantage of the General Purpose-GPU Computation CUDA library. This implementation provides approximately 2.5 orders of magnitude improvement over the existing NISE serial implementation. There are still ways that this code can be improved, both in performance and functionality. With WrightSim, we aim to lead by example among the spectroscopic community by providing an open-source package for general-purpose MDS simulation.

\section{References}

[BDH06] Paul Blanchard, Robert L Devaney, and Glen R Hall. Numerical Methods. In Differential Equations, chapter 7, pages 627-667. Thomson Brooks/Cole, third edition, 2006.

$\left[\mathrm{CTK}^{+} 15\right]$ Kyle J. Czech, Blaise J. Thompson, Schuyler Kain, Qi Ding, Melinda J. Shearer, Robert J. Hamers, Song Jin, and John C. Wright. Measurement of ultrafast excitonic dynamics of fewlayer MoS2using state-selective coherent multidimensional spectroscopy. ACS Nano, 9(12):12146-12157, dec 2015. doi: 10.1021/acsnano.5b05198.

$\left[\mathrm{FGG}^{+}\right.$09] Frederic Fournier, Rui Guo, Elizabeth M. Gardner, Paul M. Donaldson, Christian Loeffeld, Ian R. Gould, Keith R. Willison, and David R. Klug. Biological and biomedical applications of twodimensional vibrational spectroscopy: Proteomics, imaging, and structural analysis. Accounts of Chemical Research, 42(9):13221331, sep 2009. URL: https://doi.org/10.1021/ar900074p, doi : $10.1021 / \operatorname{ar} 900074 \mathrm{p}$.

[GED09] Maxim F. Gelin, Dassia Egorova, and Wolfgang Domcke. Efficient calculation of time- and frequency-resolved four-wavemixing signals. Accounts of Chemical Research, 42(9):12901298, sep 2009. URL: http://dx.doi.org/10.1021/ar900045d, doi : 10.1021 / ar $900045 \mathrm{~d}$.

[Gib02] J.W. Gibbs. Elementary Principles in Statistical Mechanics: Developed with Especial Reference to the Rational Foundations of Thermodynamics. C. Scribner's sons, 1902. URL: https: //books.google.com/books?id=IGMSAAAAIAAJ.

[Gro16] Wright Group. Nise: Numerical integration of the shrödinger equation, 2016. URL: http://github.com/wright-group/NISE.

[jif17] jiffyclub. Snakeviz, 2017. URL: http://jiffyclub.github.io/ snakeviz/.

$\left[\mathrm{KPL}^{+}{ }^{12}\right]$ Andreas Klöckner, Nicolas Pinto, Yunsup Lee, Bryan Catanzaro, Paul Ivanov, and Ahmed Fasih. PyCUDA and PyOpenCL: A scripting-based approach to GPU run-time code generation. Parallel Computing, 38(3):157-174, mar 2012 URL: http://dx.doi.org/10.1016/j.parco.2011.09.001, doi:10 . $1016 /$ j.parco.2011.09.001.

[KTW17] Daniel D. Kohler, Blaise J. Thompson, and John C. Wright. Frequency-domain coherent multidimensional spectroscopy when dephasing rivals pulsewidth. The Journal of Chemical Physics, 147(8):084202, aug 2017. URL: https://doi.org/10.1063/1. 4986069, doi:10.1063/1.4986069.

[LA85] Duckhwan Lee and Andreas C. Albrecht. A unified view of raman, resonance raman, and fluorescence spectroscopy (and their analogues in two-photon absorption. In R. J. H. Clark and R. E. Hester, editors, Advances in infrared and Raman Spectroscopy, chapter 4, pages 179-213. London; New York, 1 edition, 1985.

[NBGS08] John Nickolls, Ian Buck, Michael Garland, and Kevin Skadron. Scalable parallel programming with CUDA. Quеие, 6(2):40, mar 2008. URL: https://doi.org/10.1145/1365490.1365500, doi : 10 . $1145 / 1365490.1365500$.

[PLMZ18] Megan K. Petti, Justin P. Lomont, Michał Maj, and Martin T. Zanni. Two-dimensional spectroscopy is being used to address core scientific questions in biology and materials science. The Journal of Physical Chemistry B, 122(6):1771-1780, feb 2018 URL: https://doi.org/10.1021/acs.jpcb.7b11370, doi:10.1021/ acs. jpcb. 7b11370.

$\left[\mathrm{PRK}^{+} 09\right]$ Andrei V. Pakoulev, Mark A. Rickard, Kathryn M. Kornau, Nathan A. Mathew, Lena A. Yurs, Stephen B. Block, and John C. Wright. Mixed frequency-/time-domain coherent multidimensional spectroscopy: Research tool or potential analytical method? Accounts of Chemical Research, 42(9):1310-1321, sep 2009 doi:10.1021/ar900032g.

$\left[\mathrm{TSM}^{+}\right]$Blaise J. Thompson, Kyle F. Sunden, Darien J. Morrow, Nathan Andrew Neff-Mallon, Kyle J. Czech, Daniel D. Kohler, Tom Parker, and Rachel Swedin. Wrighttools. doi : 10.5281/ zenodo. 1198904 . 